\title{
Synchronization in Wireless Networks for Practical MIMO-OFDM Systems
}

\author{
Muhammad Khurram Kiyani ${ }^{1}$, Muhammad Usman Ahmed ${ }^{2}$, Asim Loan ${ }^{1}$ \\ ${ }^{1}$ Department of Electrical Engineering, UET, Lahore, Pakistan \\ ${ }^{2}$ Department of Computer Sciences, LUMS, Lahore, Pakistan \\ E-mail: $\{$ khurramkiyani, muahmed $\} @$ gmail.com,aloan@uet.edu.pk \\ Received February 21, 2010; revised March 24, 2010; accepted April 25, 2010
}

\begin{abstract}
In this paper a frequency offset estimation technique for Wireless Local Area and Wireless Metropolitan Area Networks is presented. For frequency offset estimation, we have applied a low-complexity frequency offset estimator for simple AWGN channels to fading channels for MIMO-OFDM systems. Simulation results have shown that the performance of the proposed estimator is better than the low complexity frequency offset estimator designed for AWGN channels.
\end{abstract}

Keywords: Synchronization, Multiple Input Multiple Output, Orthogonal Frequency Division Multiplexing, Wireless Local Area Networks, Wireless Metropolitan Area Networks, Stanford University Interim Channels

\section{Introduction}

A lot of research has been carried out in carrier frequency offset estimation for Single Input Single Output (SISO) Orthogonal Frequency Division Multiplexing (OFDM) systems but comparatively less work has been done in Multiple Input Multiple Output (MIMO) OFDM systems. In [1], timing metric for frame synchronization and frequency offset estimation in OFDM is proposed in the downlink. In [2], a coarse timing synchronization is carried out by using autocorrelation and then Carrier Frequency Offset (CFO) is estimated by performing precise autocorrelation only on samples that have been compensated for coarse timing synchronization. Both [1] and [2] have sufficiently explored OFDM but they do not incorporate MIMO. However, in [3], a novel frequency synchronization scheme is presented which uses repeated pseudo-noise training sequences to correct $\mathrm{CFO}$ in MIMO-OFDM systems. Also, in [4], integer CFO and fractional CFO are estimated for MIMO-OFDM systems through special training sequences by solving complex or real polynomial corresponding to the cost function. Although both [3] and [4] have dovetailed MIMO with OFDM systems but they lack the practicality as they have not incorporated any particular standard. In this paper we have extended the work of Luise \& Reggia- nnini (L \& R), [5], by adapting their AWGN single channel frequency estimator to multipath fading channels using IEEE 802.16-2004 Standards [6], and IEEE 802.11n Standards [7]. The technique used is non-recursive as realistic MIMO scenarios entail changing channels with information being sent in bursts corresponding to the duration of the coherence time of the channel.

The remaining paper is organized as follows. Section 2 covers the details of system model. Section 3 gives the description of the proposed frequency offset estimator and Section 4 explains the results obtained through simulations. Section 5 finally gives the conclusion.

\section{System Model}

A frequency offset can be introduced by relative motion between the transmitter and the receiver (Doppler spread) and by the inaccuracies in the Local Oscillator (LO). Channel estimation in MIMO-OFDM system is very sensitive to any frequency offset in the down converted signal because frequency offset introduces a time dependant factor that degrades the estimation of channel response matrix $H$. Therefore accurate frequency offset estimation would result in a better channel estimate and a more robust system.

Generally, a multipath fading channel is changing therefore the transmission is done in packets with the packet length being governed by the coherence time of the channel, i.e., the time for which the channel response 
does not change. In packet based communications, the channel response matrix $H$ must be estimated for each packet. This is generally done by using a training sequence known to the receiver. We have used SUI Channels, [8], here as multipath fading channels and a preamble specified in IEEE 802.16-2004 and IEEE 802.11n as a training sequence for WMAN and WLAN respectively. Our proposed algorithm estimates a constant frequency offset over a length of symbols for every packet.

In MIMO, the signal received at each receiving antenna is the superposition of the transmitted signals that from different transmit antennas. Thus, the training signal for each transmit antenna needs to be transmitted without being interfered by the others. Figure 1 shows three transmission patterns that avoid interfering with one another: independent, scattered and orthogonal patterns. For the sake of brevity we will only discuss the independent pattern. The independent pattern transmits training signal from one antenna at a time while the other antennas are silent, thus guaranteeing orthogonality, in the time domain, between each training signal. The independent pattern is often the most appropriate for MIMO-OFDM, since the preamble is usually generated in the time domain.

To encode the training sequence we have used the independent pattern and assigned to each transmit antenna a standard training sequence. This means that at a given time only one transmit antenna is transmitting the training sequence as shown in Figure 2. We have also assumed that the distance between transmitting antennas is less than $\lambda / 2$ and they all encounter the same channel statistics.

\section{Proposed Frequency Offset Estimation Technique}

The training sequence symbol is transmitted from the $m^{\text {th }}$ transmit antenna and received on any of the receive antenna. The $k$ th sampling index of this received symbol can be written as:

$$
r(k)=h_{m} c_{k} e^{j[2 \pi v k T+\theta]}+n(k) \text { for } 1 \leq k \leq N .
$$

In the above equation, $h_{m}$ denotes the complex channel coefficient between the $m$ th transmit antenna and a specific receive antenna, $v$ is the frequency offset that needs to be estimated and $n_{k}$ is the complex white Gaussian noise with variance $N_{o}$.

In general, there would be multiple receive antennas and each receive antenna would have a separate LO that would introduce some frequency drift; this means that frequency estimation needs to be done separately for each receive antenna. The frequency offset $v$ could possibly be different for different sub-carriers because Doppler induced frequency shifts depend on the wavelength of the transmission. Data modulation can be removed by multiplying $r(k)$ with $c_{k}{ }^{*}$, because for PSK constellations $c_{k} c_{k}{ }^{*}=1$ and the training sequence $z(k)$ is known(The training sequence symbols, taken from IEEE Standards 802.11n and 802.16, are assumed to be a Phase Shift Keying (PSK) constellation such as Quadrature Phase Shift Keying).

$$
z(k)=r(k) \times c_{k}^{*}=h_{m} e^{j[2 \pi v k T+\theta]}+n(k) .
$$

The autocorrelation of the $z(k)$ sequence is defined as:

$$
R(p) \equiv \frac{1}{(N+1-p)} \sum_{k=p}^{N} z(k) z^{*}(k-p) \text { for } 1 \leq k, p \leq N \text {. }
$$

Substituting (2) in (3) we get the following expression for $R(p)$ :
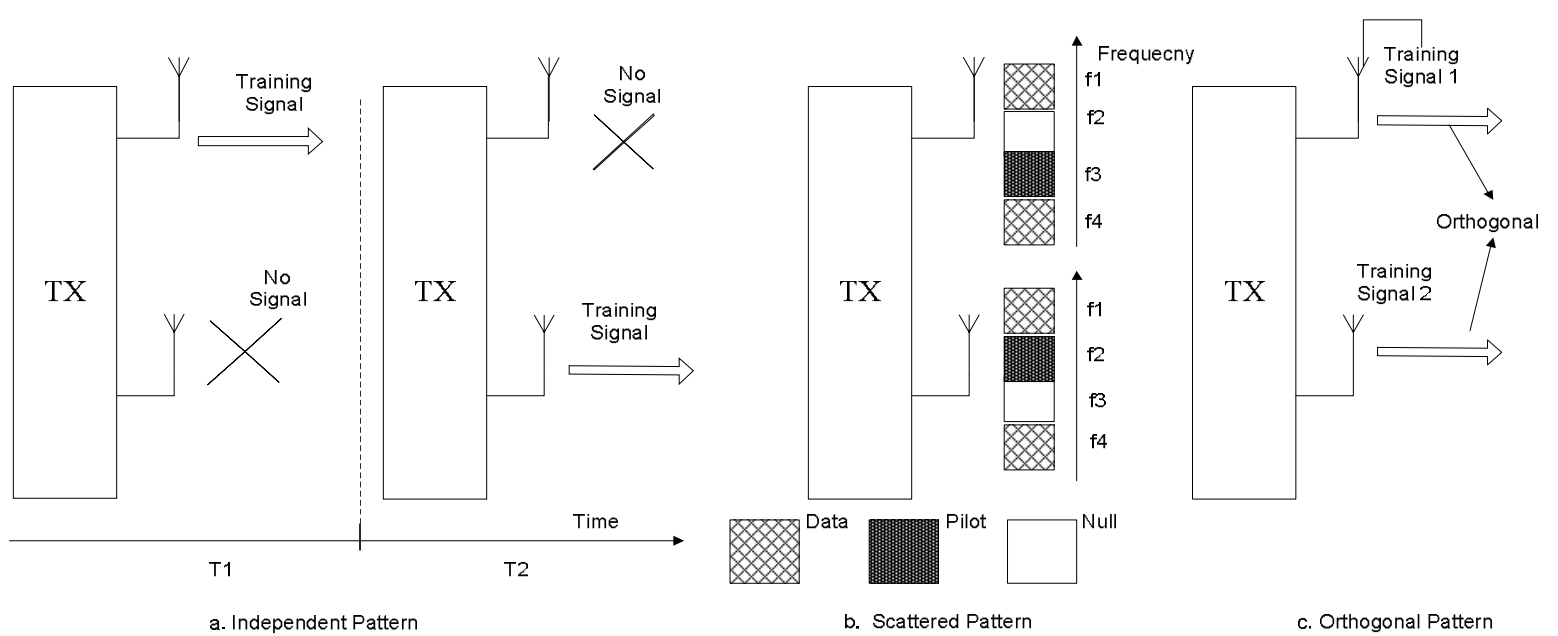

Figure 1. Three different patterns for transmitting training signals in MIMO-OFDM systems [9]. 


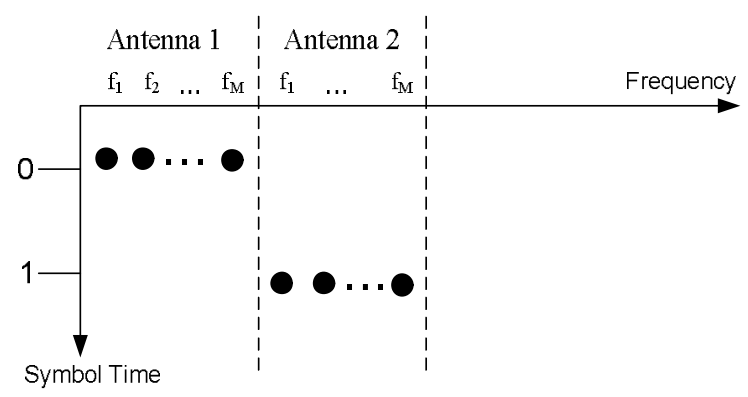

Figure 2. Space Time Encoding of the training sequence.

$$
R(p) \equiv e^{j 2 \pi p v T}+n^{\prime \prime}(p)
$$

where $\left|h_{m}\right|^{2}$ is normalized to be equal to 1 and $n^{\prime \prime}(p)$ represents the noise related(noise-noise and noise-signal) terms after substitution.

We can find a frequency offset estimate now using the formula of L\&R given below:

$$
\hat{v}=\frac{1}{\pi(N+1) T} \arg \left\{\sum_{p=1}^{N} R(p)\right\} .
$$

The summation of $R(p)$ in (3) serves to smooth out the noise as it is a moving average filter which is low pass and ideal for noise smoothing. In our MIMO-OFDM system we propose to go one step further and cross-correlate $R(p)$ with a training sequence transmitted from the second antenna in the next time slot with the same channel coefficient $h_{m}$ as shown in Figure 3.The packet length is assumed to be longer than multiple time slots and the channel remains constant over a packet length.

The cross-correlations give a greater noise-averaging gain. For this case the term within the summation in Equation $(3), z(k) z^{*}(k-p)$ is replaced by auto correlations and cross- correlations of the symbol transmitted from first and second antenna, respectively:

$$
\frac{1}{4}\left[\begin{array}{l}
z(k) z^{*}(k-p)+z(l) z^{*}(l-p)+ \\
z(k) z^{*}(l-p)+z(l) z^{*}(k-p)
\end{array}\right] .
$$

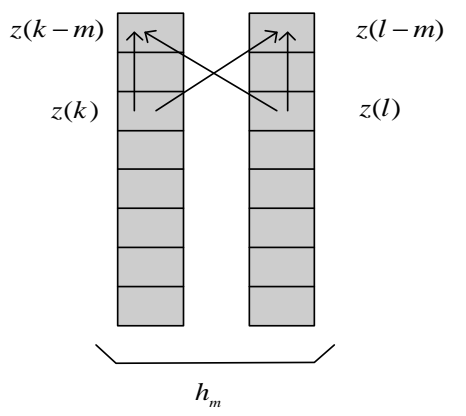

Figure 3. Auto and cross correlations of the training sequences of two transmit antennas.
For a general case of MIMO systems, $N$ transmit antennas would results in $N^{2}$ terms in our proposed correlations.

\section{Simulation Results}

Here we explore the performance of proposed frequency offset estimator for multipath fading channels using Mean Square Error (MSE) as a performance metric. We have used SUI channel 1 and SUI channel 3 as multipath fading channel for our simulations. Initially we discuss the results of WLAN and then the results of WMAN will be discussed subsequently.

For WLAN we have used $5 \mathrm{GHz}$ licensed band with nominal channel bandwidth of $20 \mathrm{MHz}$. The transmitted carrier frequency of both the base station and subscriber station should have accuracy better than $\pm 20 \times 10^{-6}$ as per IEEE Standards $802.11 \mathrm{n}$. The value should remain valid over a given temperature range and time of operation i.e., ageing of equipment. Keeping aforementioned in view the maximum carrier frequency offset comes out to be $200 \mathrm{kHz}$. A packet size of $1 \mathrm{~KB}$ is assumed. Unit delay of channel is assumed to be the same as OFDM sample period.

In Figure 4 the original curves refer to estimating the frequency offset by taking autocorrelations of the $z(k)$ sequences whereas the modified curves refer to using auto and cross correlations of $z(k)$ and $z(l)$, respectively for SUI 1 channel. The curves in this figure are generated for the cases of two transmit antennas. The modified curves, as per the proposed algorithm, provide a $2 \times \log (N)$ $\mathrm{dB}$ noise averaging gain in AWGN conditions. However, in the presence of multipath fading, it is not possible to see the complete noise averaging gain, especially for



Figure 4. Performance of the proposed estimator for SUI 1 channel. 
high $\mathrm{E}_{\mathrm{b}} / \mathrm{N}_{0}$ values. This is because signal attenuation due to fades overshadows the effects of noise.

Similarly the performance of the proposed algorithm for MIMO-OFDM system for the case of SUI 3 channel is shown in Figure 5 below. Same simulation parameters are used for both SUI 1and SUI 3 channel.

For WMAN We have used $3.5 \mathrm{GHz}$ licensed band with nominal channel bandwidth of $3.5 \mathrm{MHz}$ The transmitted carrier frequency of both the base station and subscriber station should have accuracy better than \pm 10 $\times 10^{-6}$ as per IEEE Standard $802.16 \mathrm{~d}$ and the maximum carrier frequency offset comes out to be $70 \mathrm{kHz}$. A packet size of $1 \mathrm{~KB}$ is assumed.

In Figure 6 below the original curves refer to estimat-

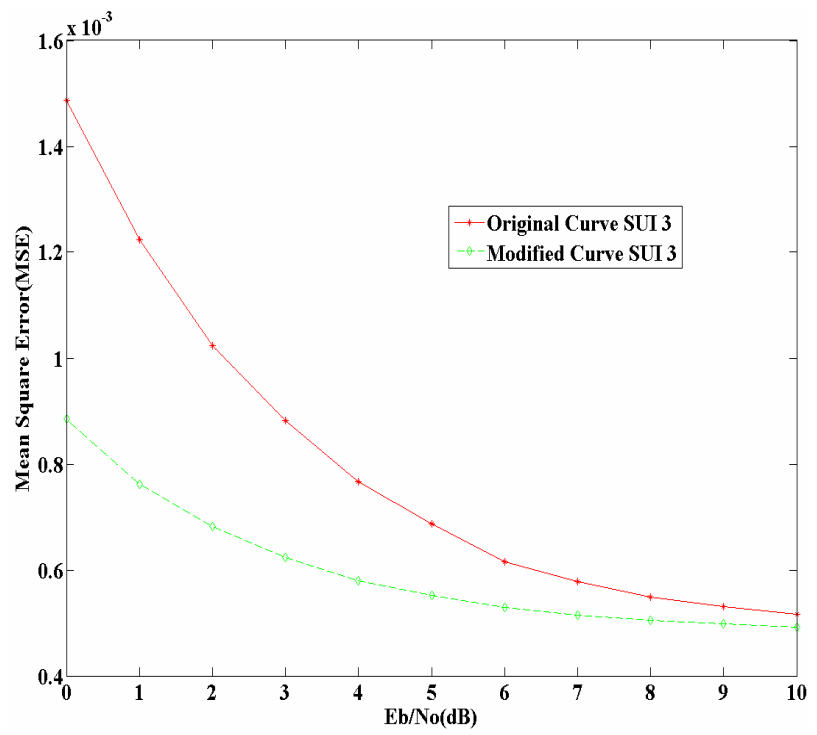

Figure 5. Performance of the proposed estimator for SUI 3 channel.

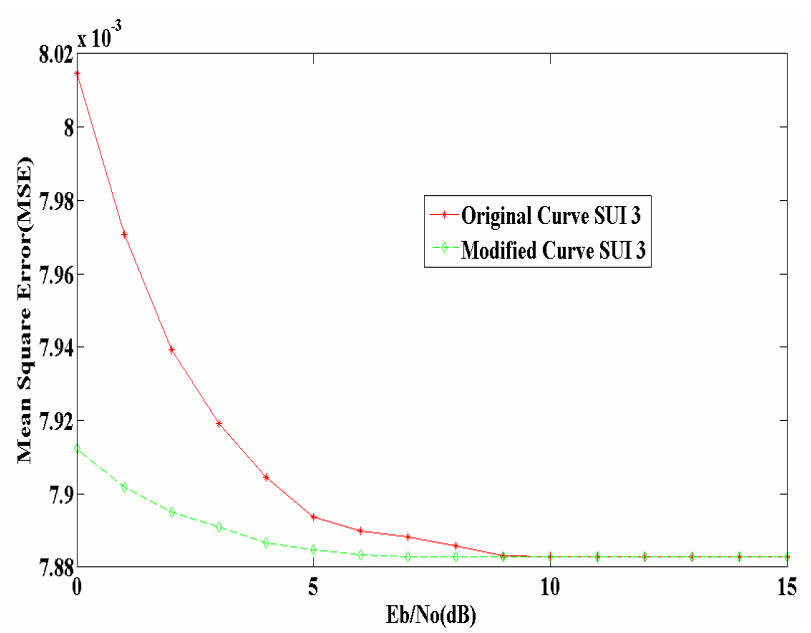

Figure 6. Performance of the proposed estimator for SUI 1 channel for WMAN. ing the frequency offset by taking autocorrelations of the $z(k)$ sequences whereas the modified curves refer to using auto and cross correlations of $z(k)$ and $z(l)$, respectively for SUI 1 channel. The curves in this figure are generated for the cases of two transmit antennas.

Similarly the performance of the proposed algorithm for MIMO-OFDM system for the case of SUI 3 channel is shown in Figure 7 below. Same simulation parameters are used for both SUI 1 and SUI 3 channels.

The salient aspects of the simulated results are analysed as under:-

1) The modified curves, as per the proposed algorithm, provide a $2 \times \log (N) \mathrm{dB}$ noise averaging gain in AWGN conditions. However, in the presence of multipath fading, it is not possible to see the complete noise averaging gain, especially for high $E_{b} / N_{0}$ values. This is because signal attenuation due to fades overshadows the effects of noise.

2) It is quite evident that the modification suggested in this paper can reduce the MSE significantly for lower values of $\mathrm{E}_{\mathrm{b}} / \mathrm{N}_{0}$. The complexity of the system may have increased but it may be traded-off for more accurate frequency offset estimation.

3) Performance of the proposed algorithm is dependent, apart from other factors, on the length of the training sequence. The training sequence used for WLAN is greater in length as compared to the training sequence of WMAN. Resultantly the results of WLAN are better as compared to WMAN especially for higher values of $\mathrm{E}_{\mathrm{b}} / \mathrm{N}_{0}$.

4) Complete execution of the proposed algorithm requires the symbols transmitted in adjacent time slots to be received at the receiver.

\section{Conclusions}

In this paper an efficient frequency offset estimation

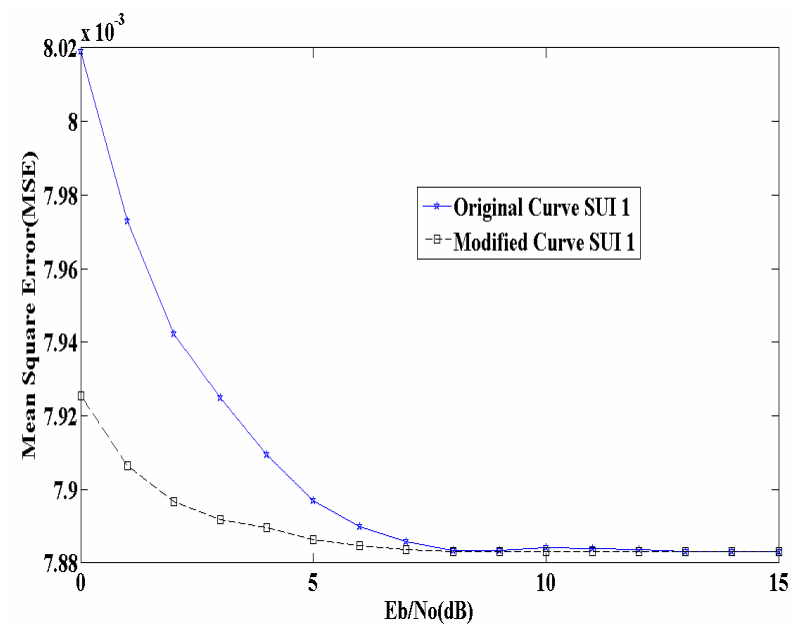

Figure 7. Performance of the proposed estimator for SUI 3 channel for WMAN. 
technique for MIMO-OFDM systems in multipath environment is presented. Simulation results have shown that synchronization problems in MIMO-OFDM systems can be solved with proposed algorithm which gives good performance and tends to be limited only by multipath fading. Since our extension of the simple L \& R estimator to the MIMO-OFDM case deals with data encoding and not with the final estimation step, we have preserved the optimality property of the L \& R estimate.

\section{References}

[1] C. N. Kishore and V. U. Reddy, "A Frame Synchronization and Frequency Offset Estimation Algorithm for OFDM System and its Analysis," EURASIP Journal on Wireless Communications and Networking, Vol. 2006, 2006, pp. 1-16.

[2] T.-H. Kim and I.-C. Park, "Two Step Approach for Coarse Time Synchronization and Frequency Offset Estimation for IEEE 802.16d Systems," IEEE Workshop on Signal Processing Systems, Shanghai, 17-19 October 2007, pp. 193-198.

[3] L.-M. He, "Carrier Frequency Offset Estimation in MIMO OFDM Systems," 4th IEEE Conference on WiCOM, Dalian, 12-14 October 2008, pp. 1-4.

[4] Y. X. Jiang, X. H. You, X. Q. Gao and H. Minn, "Train- ing Aided Frequency Offset Estimation for MIMO OFDM Systems via Polynomial Routing," 67th IEEE Vehicular Technology Conference, Singapore, 11-14 May 2008.

[5] M. K. Kiyani, M. U. Ahmed and A. Loan, "Synchronization in Fixed Broadband Wireless Access for Practical MIMO OFDM systems," 9th IEEE Malaysian International Conference on Communications, Kuala Lumpur, 15-17 December 2009.

[6] M. Luise and R. Reggiannini, "Carrier Frequency Recovery in All-Digital Modems for Burst-Mode Transmissions," IEEE Transactions on Communications, Vol. 43, No. 2-4, 1995, pp. 1169-1178.

[7] "IEEE P802.11n/D11.0 Draft Standard for Information Technology, Telecommunications and Information Exchange between Systems," Local and Metropolitan Area Networks-Part 11: Wireless LAN Medium Access Control (MAC) and Physical Layer (PHY) Specifications, 2009.

[8] K. V. Erceg, S. Hari, M. S. Smith, D. S. Baum, et al., "Channel Models for Fixed Wireless Applications," IEEE 802.16.3 Task Group Contributions, 1 February 2001.

[9] G. J. Andrews, A. Ghosh and R. Muhamed, "Fundamentals of WiMAX Understanding Broadband Wireless Networking," Prentice Hall Communications Engineering and Emerging Technologies Series, Prentice Hall, February, 2007. 